\title{
Expiration flow in a symmetric bifurcation
}

\author{
F.E. Fresconi, A.S. Wexler, A.K. Prasad
}

\begin{abstract}
This work investigates the fundamental flow in a converging bifurcation. Particle image velocimetry (PIV) and laser-induced fluorescence (LIF) experiments were conducted in a transparent model composed of three machined tubes mated together in a Y-shape. Measurements were taken in a plane containing the axes of the tubes and in the cross-sectional plane of the parent tube to elucidate flow patterns in the bifurcating passages of the human respiratory system. The primary objective was to determine the amount of secondary flow in a bifurcation during expiration. PIV measurements in the transverse plane show detailed characteristics of the secondary flow. Results at higher $R e$ illustrate unsteadiness associated with a hairpin vortex and symmetry-breaking. This study addresses the transport of particles of small Stokes number that survive inertial impaction during inhalation and remain in suspension over multiple breathing cycles. Knowledge of the underlying airflow is required to develop an accurate dosimetry model to transport prescription drugs with precision into the bloodstream. Similarly, the harmful impact of pollutants on the respiratory system can only be assessed by first understanding the carrier airflow.
\end{abstract}

\section{1}

Introduction

\section{1}

\section{Motivation}

The flow in a converging bifurcation has widespread engineering and biological applications. Perhaps the most important application is the flow in a human lung. The

Received: 15 April 2003 / Accepted: 1 September 2003

Published online: 11 October 2003

(c) Springer-Verlag 2003

\section{F.E. Fresconi ( $\square)$, A.K. Prasad}

Department of Mechanical Engineering,

University of Delaware, Newark, DE 19716, USA

E-mail: fresconi@me.udel.edu

Tel.: +1-302-8312960

Fax: +1-302-8313691

\section{A.S. Wexler}

Department of Mechanical and Aeronautical Engineering,

Department of Civil and Environmental Engineering,

Department of Land, Air and Water Resources,

University of California, Davis, CA 95616-5294, USA

Research described in this article was supported by Philip Morris Incorporated. human airways are a complicated series of bifurcating tubes. From the trachea, the network splits into the two main bronchi and continues to bifurcate throughout the conducting and pulmonary regions until the final alveoli are reached. Lung models characterize this branching into about 24 generations. The Reynolds number $(R e)$ in this network ranges from about 10,000 at the trachea to less than 0.01 in the alveolar region, depending on breathing conditions. An investigation into the flow in the human lung is further complicated by compliant walls and a thin lining of mucus. Upon inhalation, a bolus of particles will experience an axial stretching. Particles of small Stokes number that survive inertial impaction during inhalation will experience cross-sectional mixing due to secondary currents on expiration. These irreversible processes do not permit the original bolus shape to reassemble after one cycle. This implies that some part of the bolus will remain suspended in the lung and penetrate deeper over multiple breathing cycles. If the complex flow in this geometry can be understood, it can be coupled with the transport and deposition of particles and gases entering the lung. Medical delivery systems, such as metered dose inhalers, and pollution effect studies would profit greatly from such an understanding.

\section{2}

\section{Past work}

Some of the earliest experimental work on a bifurcating tube model was by Schroter and Sudlow (1969). They were the first to present many of the basic features, such as the secondary vortices and the shape of the longitudinal velocity profiles. Secondary vortices (called Dean vortices) arise as a result of the Dean effect, which is a laminar centrifugal instability that can occur when a viscous fluid flows in a curved duct with an axial pressure gradient (Drazin and Reid 1981). This phenomenon is due to the inability of pressure to counterbalance the centrifugal force at a given cross-section. In the plane containing the radius of curvature, flow proceeds from the inner to the outer wall of curvature. At the outer wall, the flow must turn and follow the circumference of the tube until the inner wall is again reached. This results in two secondary vortices mirrored about the plane of symmetry. Olson (1971) has also made some pioneering contributions consisting of assumptions that the flow can be considered quasi-steady and that the deformation of the walls can be neglected for normal breathing rates. This allows the use of a model with steady inlet flow and rigid boundaries. 
Kim et al. (1994) and Kim and Garcia (1991) used different glass tube models to investigate the effect of many different parameters on the flow and deposition in the lung. These variables included branch symmetry, branch plane, branch angle, compliant wall, liquid lining, Stokes number $(S t)$, diameter ratio, cyclic frequency and $R e$. Many parameters, such as the diameter ratio, should be negligible after illustrating the relative impact of different terms, and the deposition efficiency becomes a function only of Stokes number. This work has shown that a rather simple model can be robust enough to provide insight into the physics at a bifurcation.

Although the geometry was not physiologically representative, work done by Zhao and Lieber (1994) has become the current experimental benchmark. LaserDoppler velocimetry (LDV) was used in a three-generation model to obtain axial velocity profiles on inspiration and expiration that were more detailed than those obtained by Schroter and Sudlow (1969). Their results show that expiration is less prone to turbulence than inspiration is, due to the stabilizing effect of the area constriction and secondary vortices. Also, no separation was detected on inspiration. The highest secondary flow was measured at the bifurcation, with a decrease in strength downstream.

Computational investigations into the flow have recently been fruitful. Modeling by Comer et al. (2001), Zhang and Kleinstreuer (2002), and Zhang et al. (2001) has shown that three features affect the flow in a bifurcation: upstream flow, Dean effect, and carinal ridge shape. Comer et al. (2001) also argued that the steady flow shows the upstream affects are felt downstream but not vice versa to the same degree.

\section{3}

\section{Present goal}

The objective of this study is to understand the secondary flow in a converging bifurcation model. Secondary motion is responsible for particle dispersion in the human airways (Sarangapani and Wexler 1999). An understanding of secondary currents on expiration is especially useful since these are relatively stronger and therefore play a more crucial role. PIV and LIF are used to characterize the flow within a simple model. These results provide insight into the transport of gas and particles which remain suspended in the airways after inspiration. Future models can extend on fundamental principles of this work.

\section{2}

\section{Experimental model and methods}

\section{1}

\section{Lung morphometry}

The geometry of the lung is difficult to characterize. Much work has been done (Weibel 1963; Horsfield et al. 1971) to determine statistics, but the system can still feature much variability. For this reason, it is not desirable to reproduce in a physical model all the possible geometries. The approach in this work is to begin with a simple model. Future efforts will focus on more anatomically accurate geometries.
A single generation model was considered in this study. All tubes were of equal diameter: $2.86 \mathrm{~cm}$. While this allowed ease of machining, this can be considered a departure from reality. Most bifurcations in the conducting region of the human lung have an area ratio of about 1.2 from the parent to the daughter branches (Pedley 1977). Despite this drawback, the model still presents the fundamental flow features typical of the lung. Some work (Kim et al. 1994) has even shown a negligible effect of area ratio on flow characteristics such as deposition. The consequence of this idealized geometry will be an acceleration of the flow as the two daughter branches converge into the parent tube, resulting in a more stabilized flow. The halfangle between daughter tubes was $30^{\circ}$, typical of most bifurcations in the central airways. The length to diameter ratio of the tubes was 4 , close to the value of 3.5 given in the literature (Horsfield et al. 1971). The radius of curvature for the model was estimated as $0.1 \mathrm{~m}$. The radius of curvature in the lung is one of the physiological parameters that is extremely difficult to accurately describe and can feature much variability, depending on generation. Our model has rather sharp edges in the carinal area, but this is still not significantly different from the $r / d=0.1$ value quoted in Kim et al. (1994), where $r$ is the radius of the carina and $d$ is the branch diameter. It has also been shown (Comer et al. 2001) that the carina shape only affects the flow in the region near the carina. A limitation of this model is the abrupt transition region from the parent to the daughter branches. As described in Horsfield et al. (1971), proceeding from the parent to the daughter branches, a cross-section will evolve from circular to elliptical until it begins to kink at the minor axis and eventually morph into two circles. The wide range of anatomical variability encountered in nature, however, ensures that some bifurcations are more abrupt. Some anatomical features, such as the elasticity of the lumen and thin lining of mucus, are not considered in this model. The geometrical information presented is typical of the conducting airways (Pedley, 1977).

\section{2}

\section{Experimental setup}

The bifurcation model was fabricated out of Plexiglas due to its transparency and ease of machining. A fluid-filled box was built around the test section to enable refractive index matching. The working fluid was water. A large reservoir was situated upstream of the daughter tubes. Flexible tubing with valves connected the test section to the reservoir. The parent tube was connected using similar tubing to a large piston/cylinder arrangement. The piston was driven by a stepper motor. The stepper motor controller allowed the piston movement to be programmed (see Fig. 1).

The general area of the lung to which this model may be applied is the conducting region. This approximately constitutes the first 17 generations past the main bronchi. The present model represents idealized geometry which does not completely capture the complex physiological anatomy; future studies will explore the effect of more realistic geometry on the flow. Reynolds numbers in this area for typical breathing rates range from 0.1 to 2,400 


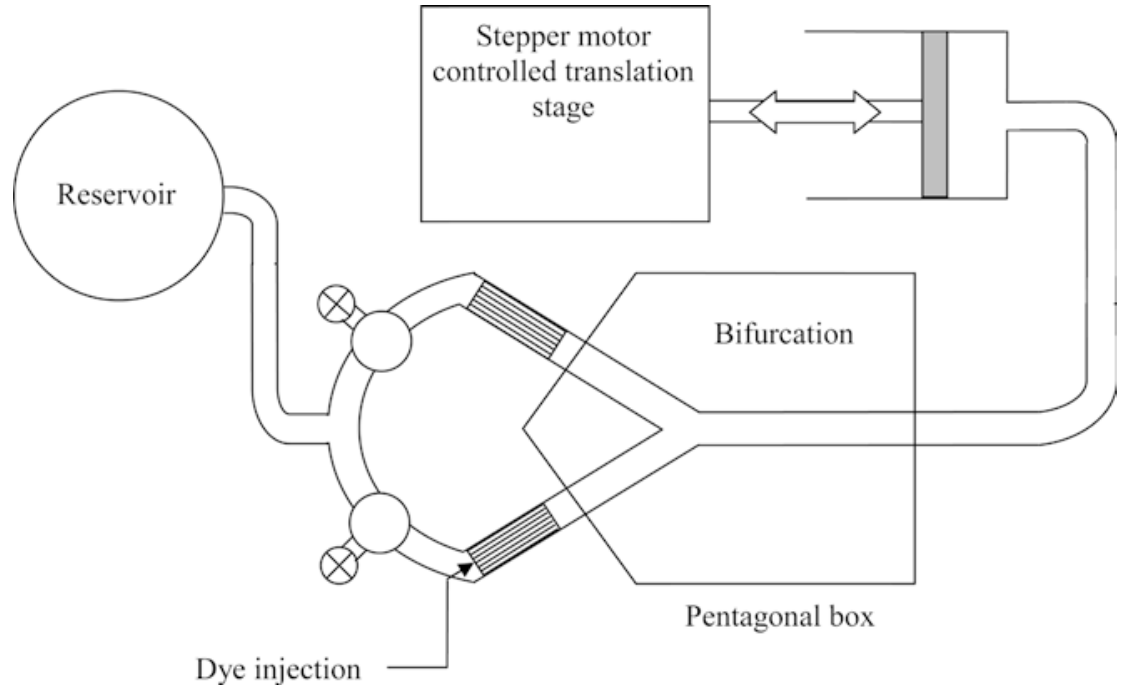

495

Fig. 1. Experimental apparatus
(Pedley 1977). Re for our study ranges from 680 to 1,380 . Corresponding Dean numbers $\left[D n=\operatorname{Re}(d / R)^{1 / 2}\right]$ are 260-520, where $d$ is the diameter and $R$ is the radius of curvature.

Two important planes must be defined at this point. The plane containing the bifurcation (i.e., the plane containing the axes of the daughter branches and parent tube) will be denoted as the bifurcation plane. The plane normal to the bifurcation plane and containing the axis of the parent tube will be denoted as the normal plane.

\section{3}

\section{Flow considerations}

All experiments were performed with a steady inlet flow. As discussed previously, a quasi-steady assumption is reasonable for normal breathing rates. Obviously, the inlet flow into any branch in the lung is the outlet flow from the previous branch. For expiration the inlet flow will have four secondary vortices due to the junction, along with residual vorticity from previous bifurcations. This will serve to flatten out the usual parabolic profile in a laminar straight pipe flow, similar to the effect of turbulence. Also dependent on the generation (and therefore $R e$ ) is the entrance length effect. Bifurcations closer to the trachea have a higher $R e$ and therefore longer development length. In any case, centrifugal effects just upstream of the junction will result in skewing of the profile toward the wall furthest from the center of curvature (Zhao and Lieber 1994; Zhang and Kleinstreuer 2002). In this model, the flow is conditioned by passing it through a length of tube packed with straws before reaching the test section. This ensures that any large-scale eddies are eliminated and the flow streamlines are parallel. Now, the distance from the flow conditioners to the measurement volume is less than the hydrodynamic entry length. This gives us a laminar, flattened velocity profile similar to that actually found in the lung (Zhang and Kleinstreuer 2002).

\section{4}

\section{Measurement techniques}

LIF and PIV measurements were conducted with the model. The choice of circular geometry for the flow passage can lead to severe optical distortions during flow imaging unless remedial measures are undertaken. This is especially true during longitudinal imaging (i.e., in an axial plane). Distortions were reduced by refractive index matching: a transparent pentagonal box with flat faces enclosed the bifurcation, and this box was filled with water. The refractive index of water (1.33) does not exactly match that of the acrylic model $(\approx 1.5)$ (see Fig. 1 ), therefore a simple test was performed to assess the significance of this mismatch in refractive indices. An acrylic tube of exactly the same diameter and thickness as the model tubes was halved by cutting it lengthwise along the diameter. This half-tube was placed convex side up in the pentagonal box at the exact height of the model. A transparency printed with a Cartesian grid was taped to the axial plane of this specially prepared tube. The box was then filled with water and imaged from above. Figure 2 shows the transparent half-tube in the test section with the grid beneath. Notice how little the horizontal lines of the grid are disturbed by the presence of the acrylic tube. The image was digitized and analyzed to determine the extent of optical distortion. Even near the tube wall (where lensing effects would be greatest), the distortion was acceptably small (less than 5\%). For the cross-sectional experiments a special, orthogonal viewport allowed the flow to be imaged in a "head-on" manner so that distortions due to lensing did not occur.

LIF is a visualization technique that exploits fluorescence produced when laser illumination at a specific wavelength excites dye molecules that have been added to the flow. A small quantity of Rhodamine $6 \mathrm{G}$ dye was added to the working fluid, and a Nd:YAG laser was used for illumination $(532 \mathrm{~nm})$ to excite the fluorescence. The laser sheet was $1 \mathrm{~mm}$ thick. A high-resolution $(1,300 \times 1,030$ pixels) DVC-1300C digital camera was used for LIF. A motorized syringe carefully injected dye so that the injection velocity of the dye equaled the local flow velocity. Flexible tubing connected the syringe to a dye needle placed upstream of the test section and flow conditioners. The dye needle was threaded into an individual flow conditioner straw (see Fig. 1) so that the evolution of the flow from a particular injection site could be witnessed. 


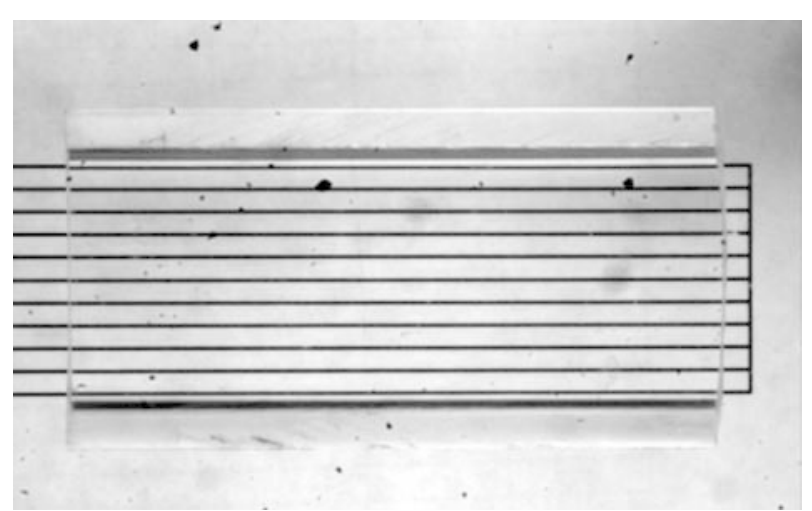

Fig. 2. Refraction for longitudinal experiments

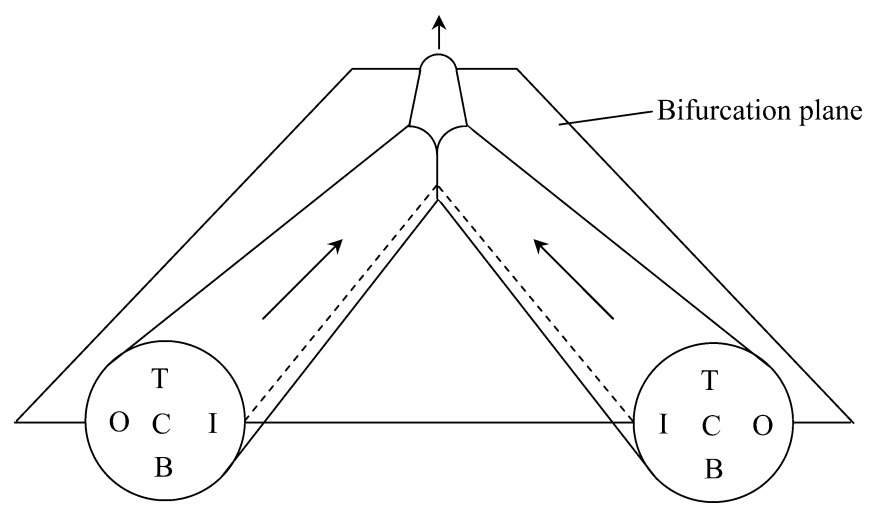

Fig. 3. Injection sites for LIF: $O$ outside; $I$ inside; $C$ center; $B$ bottom; $T$ top

Five positions in the cross-section, denoted as outside, inside, center, bottom, and top, were selected for LIF experiments. Figure 3 illustrates the labeling for injection sites. The diffusivity of Rhodamine $6 \mathrm{G}$ in water is sufficiently small to ensure that dye transport in the model was purely due to convective effects.

PIV is a well-known technique for obtaining global velocity information, instantaneously and with high accuracy. In these experiments, planar slices of the flow field that contained the axis of the pipe (longitudinal section) and the cross-section of the pipe were illuminated. The camera viewed the illuminated plane from an orthogonal direction and recorded particle images at two successive instants in time in order to extract the velocity over the planar two-dimensional domain. Hollow glass spheres with a $40 \mu \mathrm{m}$ diameter were used to seed the flow.

\section{3}

\section{Results}

\section{1}

\section{Longitudinal LIF}

Figures 4 and 5 each show LIF images of the parent airway for different injection sites (see Fig. 3 for nomenclature) and for different Reynolds numbers. A schematic is provided on the right of the LIF images to illustrate the geometry being visualized. The junction between the two daughter tubes and the parent tube is situated at the top of

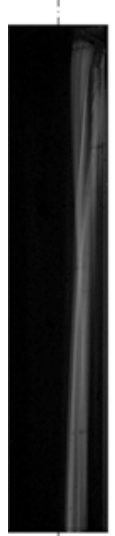

Outside

(a)

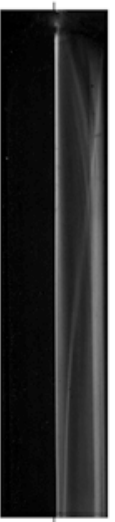

Inside

(b)

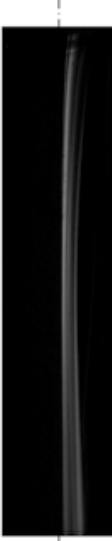

Center

(c)

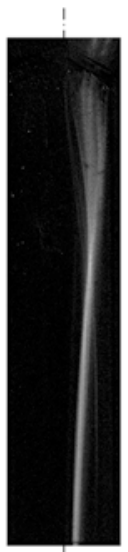

Bottom

(d)

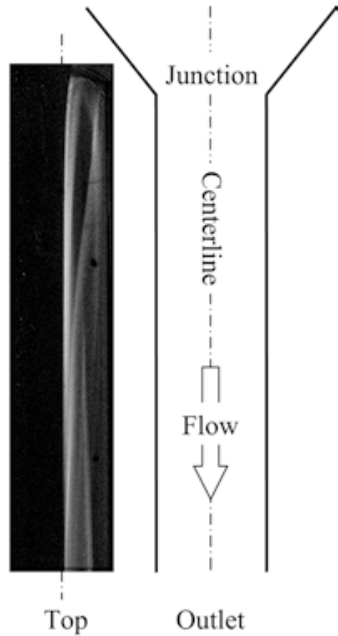

(e)

(f)

Fig. 4. Longitudinal LIF: $R e=850$

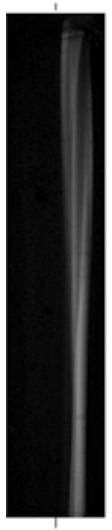

$\operatorname{Re}=680$

(a)

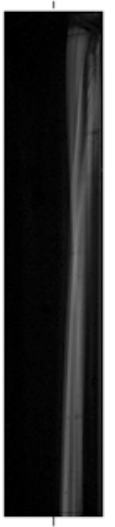

$\mathrm{Re}=850$

(b)

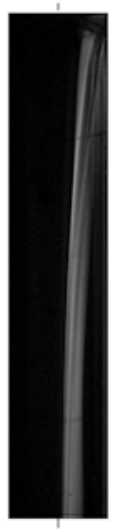

$\operatorname{Re}=1020$

(c)

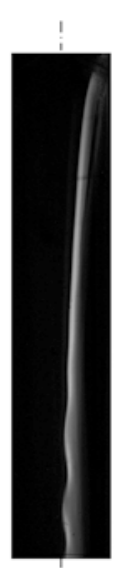

$\operatorname{Re}=1200$

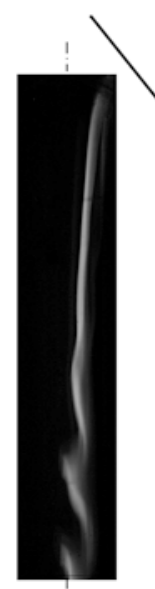

$\operatorname{Re}=1380$

(e)

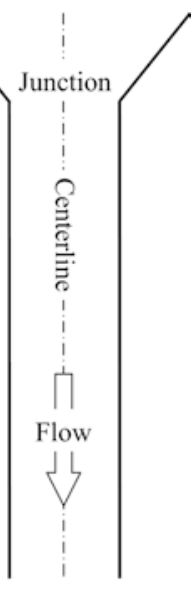

Outlet

(f)

Fig. 5. Longitudinal LIF: outside injection

each image. The flow proceeds from the daughter tubes into the parent tube (top to bottom in the figures). Dye was injected upstream into only one of the daughter branches to capture the flow patterns more clearly. Since the width of the images exactly spans the diameter, the LIF clearly shows the dyed and undyed halves of the parent tube for a length four diameters downstream of the junction.

At a given $R e$, some observations can be made in Fig. 4 concerning the streamlines of dye injected at different positions. First, the twisting of the dye stream in nearly all realizations clearly indicates the secondary flow. As the dye enters the parent tube, however, this "corkscrewing" is more or less evident, depending on the injection origin. The outside, bottom, and top injections show moderate spiraling and radial spread. Dye injected at the inside of the daughter branch indicates strong dispersion along the radius. As $R e$ increased, the dye could be seen to disperse along the radius even upstream of the junction because the secondary effect due to the bifurcating geometry is felt upstream. It was noticed that for, lower $R e$, the dye is 
constrained to that side of the flow passage where it was originally injected. However, for $R e=1,380$, the dye crosses over to the opposite side of the parent tube, breaking symmetry. The center injection is interesting in its quiescence. All injection sites except the central one reveal some degree of helical motion .

Figure 5 shows the entire $R e$ range for a particular injection site (outside), allowing us to inspect the evolution of the flow as $R e$ increases. For $R e=680-1,020$, a common thread is the corkscrewing of the dye. At $R e=1,200$, a distinct sinuous motion manifests itself some distance downstream from the bifurcation for most injection positions. The signature of an instability other than the Dean effect is beginning to gain prominence. As $R e$ increases to 1,380 , the dye breaks into a choppier, wave-like pattern, which still occurs regularly.

This instability is illustrated in Fig. 6 . Let the outside edge at the bifurcation plane be defined as the "corner" of the entrance to the parent tube. At the corner, the primary and secondary flows act within this geometry to produce an interesting formation. A long horseshoe or hairpin characterizes the shape of this feature. The narrow, open end of the hairpin is at the corner, while the rounded portion lies downstream. A plane parallel to the normal plane contains the transverse span of the hairpin instability. The hairpin grows from the corner for a length of about two or three tube diameters downstream before detaching and propagating downstream. This instability is superimposed or coupled with the Dean effect. As evident in the longitudinal LIF, this instability begins to set in at $R e=1,020$ and is well formed by $R e=1,380$. While this effect may not be as pronounced at all airway bifurcations, the physiological geometry surely features enough variability to create such an effect on occasion.

\section{2}

\section{Cross-sectional LIF}

At a longitudinal position of $x / D=1$, LIF measurements were taken over the range of $R e$ for outside and inside injections. Figure 7 shows the cross-section of the parent tube, looking head-on toward the junction (flow coming out of the page). As in the case of the longitudinal LIF, dye was injected into a single daughter branch. While the lowest $R e$ shows little spread of the dye, increasing $R e$ begins to illustrate the secondary motion. A significant amount of twisting is evident for $R e=1,020-1,380$ as the dye assumes a reverse $\mathrm{C}$-shape from being trapped within two of the quadruple vortices. This pattern captures the roll-up of the secondary vortices well. Since the dye pattern does not completely close upon itself (a reverse B-shape), it can be deduced that the secondary motion does not perform a complete revolution one diameter downstream of the junction.

\section{3}

\section{Longitudinal PIV}

PIV measurements were performed along the axis of the parent tube in the bifurcation plane for a distance of four diameters. Measurement resolution was $100 \mu \mathrm{m} /$ pixel. The interrogation box size used for PIV processing was $64 \times 64$ pixels with a $50 \%$ overlap. To obtain a clean velocity field, after the images were correlated the resulting vectors were first processed to remove spurious vectors and then averaged over fifty realizations. The relative standard deviation of the particle displacement was $9 \%$, arising primarily from flow unsteadiness. Using the standard formula for 20 -to- 1 odds, $\pm(1.96 \sigma) / \sqrt{ } \mathrm{N}$, where $\sigma$ is the standard deviation, and $N$ is the number of samples, the uncertainty in longitudinal PIV measurements is about $3 \%$. It should be noted that, while the frequency of the unsteadiness associated with the hairpin vortex was around $0.1-1 \mathrm{~Hz}$, the sampling frequency was $10 \mathrm{~Hz}$. Thus, the sampling rate was high enough to resolve the periodic motions. Figure 8 shows the velocity profiles at $x /$ $D=0,1,2,3$, and 4 . The radial direction was normalized by the tube radius, and the horizontal boundaries of each subplot denote the position of the inner wall of the pipe. The velocity field data do not extend all the way to the pipe walls due to experimental limitations. Obviously, a no-slip condition is present at the wall. A characteristic of flow in a bifurcation is that the boundary layer is thinner than that found in a fully developed straight tube flow (Olson 1971). Therefore, it can be expected that the velocities shown in Fig. 8 would extend relatively close to the wall at a similar magnitude before quickly falling to zero at the boundary. Flow direction is left to right, and a reference vector gives a sense of velocity magnitude.

Figure 8 shows that even for low $R e$ the flow is still not fully developed after $x / D=4$, which is also presented in the literature (Zhao and Lieber, 1994). At most Re, the velocity

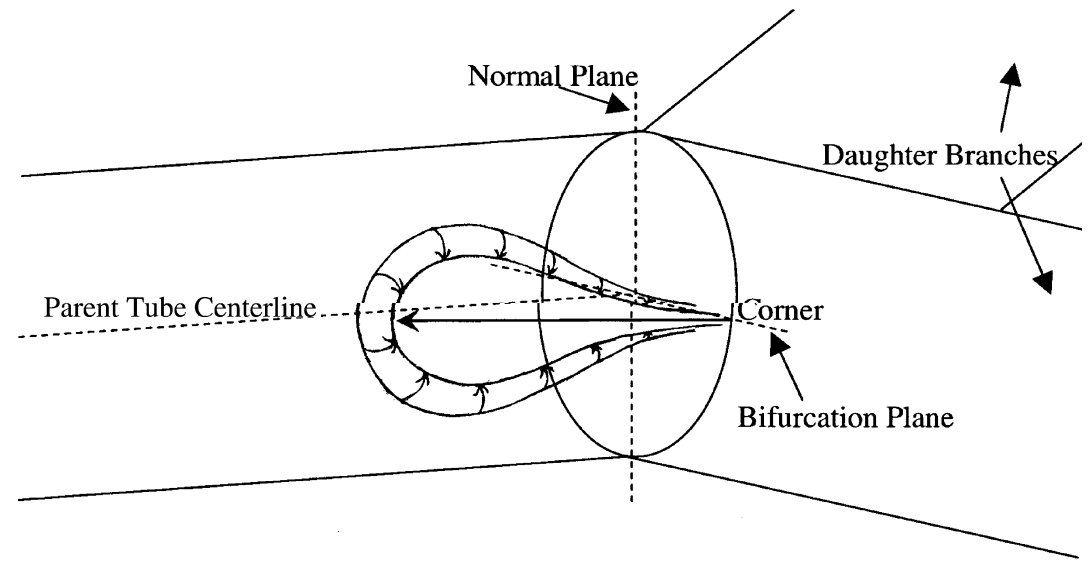

Fig. 6. Schematic of the hairpin vortex formation 

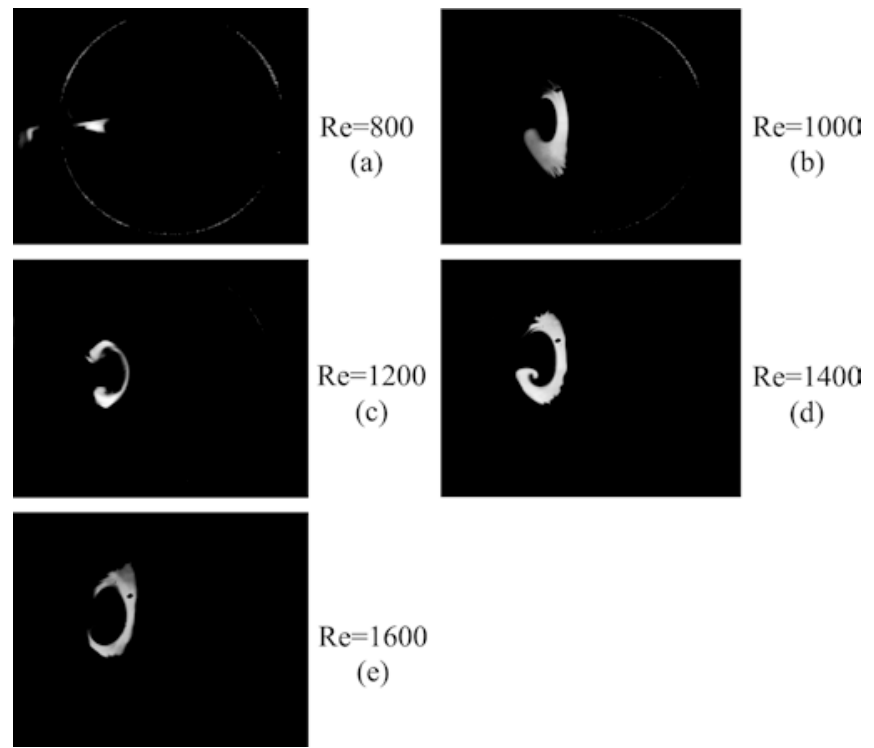

$\operatorname{Re}=1600$

(e)

Fig. 7. Cross-Sectional LIF: outside injection at $x / D=1$

profile enters the parent tube as some form of a double peak. This double peak develops into a parabolic-like shape for $R e=680$. For moderate $R e$, a weak double-peaked profile evolves into a bell shape (see $R e=850, x / D=2$, $R e=1020, x / D=1,2)$. The main feature of this bell shape is a peak at the centerline of the parent tube surrounded by inflection points on either side. Past work (Schroter and Sudlow 1969; Zhao and Lieber 1994) has shown this bellshaped profile for expiratory flow, however, they documented a more significant peak. The primary reason for the maximum in the bell-shaped profile is the radial influx of velocity along the bifurcation plane due to the secondary motion. As will be presented shortly, secondary velocity measurements are relatively low. This, along with fluctuations from the hairpin vortex, would result in a less pronounced bell-shaped profile. The distinct doublepeaked profile (see $R e=1,200, x / D=1, R e=1,380, x / D=2,3$ ) extends downstream at the higher $R e$ before changing into a more flattened profile. While most of the velocity at all cross-sections is in the longitudinal direction, the largest radial component can be attributed to $x / D=0$. Here a radial inrush is especially evident for lower Re. Also, some measurements feature a degree of asymmetry.

\section{4}

\section{Cross-sectional PIV}

PIV experiments undertaken in the cross-section of the parent tube were rather difficult. Many unfavorable circumstances are encountered as a particle-laden flow is imaged through a large depth of fluid. For example, light scattered by the illuminated particles is severely attenuated by the other particles that lie between the measurement plane and the camera. Optical distortions that arise due to the large depth of fluid are also very severe. In addition, care must be taken to ensure that the particles seeding the flow do not exit the measurement plane between illuminating pulses. For this reason, the laser sheet was expanded to a thickness of about $1 \mathrm{~cm}$. Spatial resolution of the measurements was $20 \mu \mathrm{m} /$ pixel. The interrogation box size used for PIV processing was $64 \times 64$ pixels with a $50 \%$ overlap. An average of sixty PIV frames was taken for the higher $R e$, while thirty samples were found to be adequate for lower $R e$. The uncertainty in cross-sectional PIV was less than $4 \%$. A schematic of the geometry is provided on the top-right of the figures.

The quadruple vortex is prominent in Figs. 9 and 10. A radial influx in the bifurcation plane (oriented vertically in the figures) becomes a radial outflux in the normal plane after the flow reaches the parent tube center. As the flow meets the wall (in the normal plane), it begins to traverse the circumference of the tube until it again reaches the bifurcation plane and repeats the motion. A slight asymmetry about the bifurcation plane can be seen for all crosssectional PIV. This asymmetry is not expected for ensemble-averaged vector fields; instead, it is probably a result of the unfavorable conditions during PIV processing in the cross-sectional view.

The downstream evolution of the flow for a given $R e$ is illustrated in Fig. 9. It is evident that the quadruple vortices are not yet fully rolled up at $x / D=0$. The vortex core can be seen to migrate toward the centerline as the flow proceeds downstream. This gives a sense of how quickly the secondary flow develops. Maximum secondary flow occurs at the junction, indicating the importance of this position in mixing and deposition of particles. The secondary flow then decreases as $x / D$ increases, especially for low $R e$. In contrast to previous work (Comer et al. 2001), higher $R e$ exhibits a relatively low secondary velocity throughout. Possible reasons for the difference include unsteadiness from the hairpin vortex or the difference in geometry in past studies. The acceleration effect of the area ratio may delay flow development and magnify the primary flow at the expense of secondary flow.

The data shown in Fig. 9 are rearranged in Fig. 10 to show how the secondary flow changes with $R e$. Higher Re forces the vortex cores further away from the centerline as the primary flow is more dominant and has a longer development length. Figure 10 also shows that the strength of the secondary flow is greater for the lower $R e$. The apparent breakdown of the results for high $R e$ could be due to unsteadiness and mixing from the hairpin vortex which reduces the coherence of the secondary vortices, or simply low secondary velocity. Although no previous work has performed PIV in the cross-section of such a geometry, the results compare well with the numerical work of Zhang and Kleinstreuer (2002). Their data have a similar quadruple vortex shape. However, their normalized secondary velocity strengths are around $15 \%$ higher.

\section{5}

\section{Flow development}

Some analysis was performed to summarize the evolution of the flow more concisely. A maximum value was found for secondary, longitudinal, and radial velocities. The maximum secondary velocity was found from the crosssectional PIV data for each $R e$ at every cross-section using a relation of the form, $U_{s, \max }=\max \sqrt{ }\left(u^{2}+v^{2}\right)$, where $u$ and $v$ are the $x$ and $y$ components of velocity, respectively.

Longitudinal PIV results were used to find the maximum longitudinal and radial velocity simply as, $U_{1, \max }=\max \left|U_{1}\right|$ 


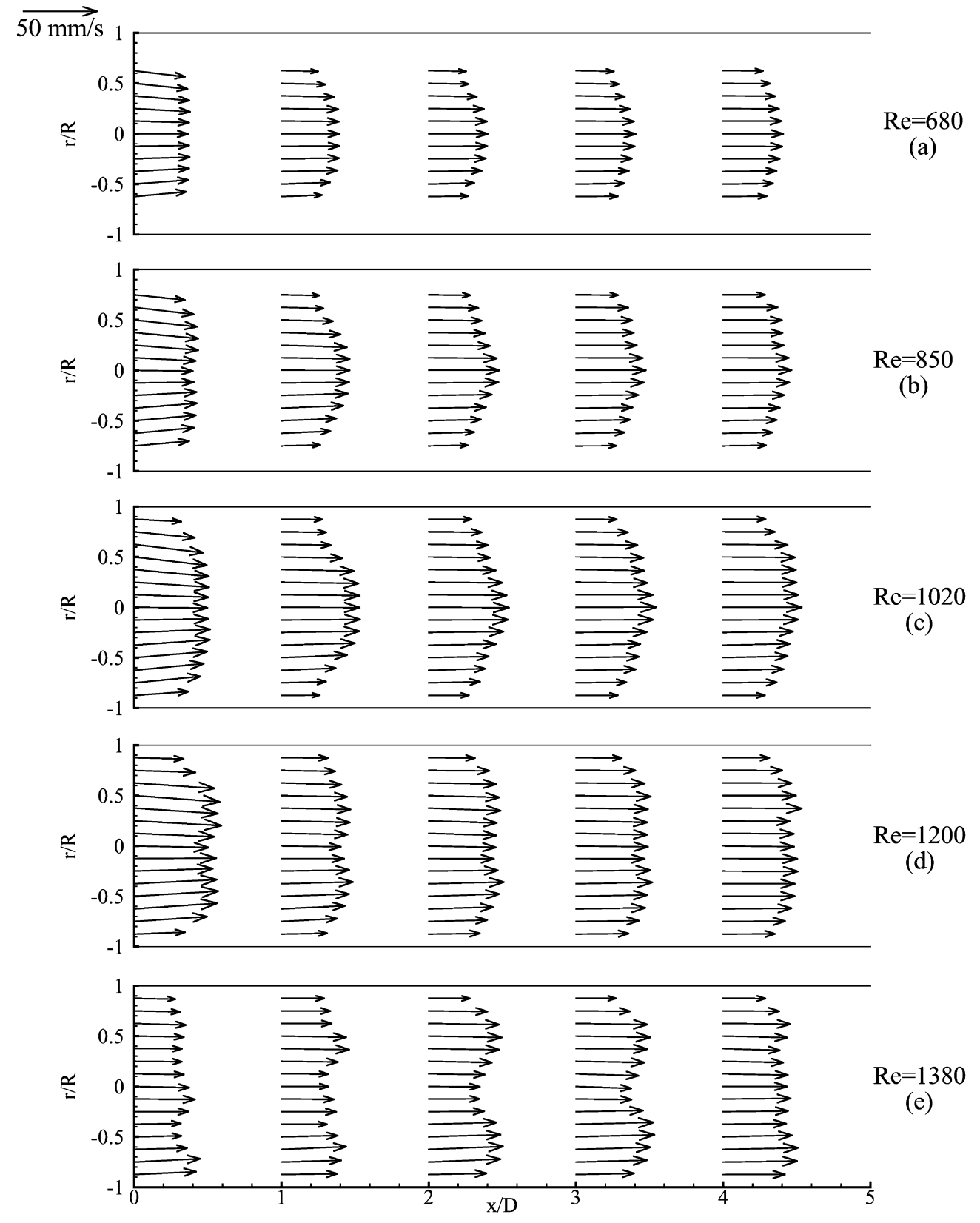

Fig. 8. Longitudinal PIV

and $U_{\mathrm{r}, \max }=\max \left|U_{\mathrm{r}}\right|$, where $U_{1}$ and $U_{\mathrm{r}}$ are the longitudinal and radial velocities, respectively. The maximum values were normalized by the average longitudinal velocity, $U_{1, \text { avg }}$, at a given $R e$. The average longitudinal velocity was calculated by dividing the volume flow rate $Q$ through the parent tube $(Q$ is controlled precisely by the stepper motor that drives the piston) by the parent tube area. Resulting normalized velocities are defined as

$U_{\mathrm{s}}^{*}=U_{\mathrm{s}, \max } / U_{\mathrm{l}, \mathrm{avg}}$

$U_{1}^{*}=U_{1, \max } / U_{1, \mathrm{avg}}$

$U_{\mathrm{r}}^{*}=U_{\mathrm{r}, \max } / U_{\mathrm{l}, \mathrm{avg}}$

The normalized maximum secondary velocity is shown in Fig. 11. A monotonic decrease with increasing $x / D$ characterizes the trend for low $R e$. Mid-range $R e$ features a slight drop in secondary strength before leveling to constant values, while the high $R e$ values remain relatively constant. It is expected that $U_{s}^{*} \rightarrow 0$ when $x / D \rightarrow \infty$. A substantial difference in $U_{s}^{*}$ exists between the low and high $R e$ at a given downstream location. As the crosssectional PIV shows, contrary to expectation the secondary velocity magnitude and $R e$ appear to be inversely related. Possible reasons for this phenomenon are the acceleration effect or the unsteady hairpin vortex. Flow acceleration would delay flow development (and therefore possibly secondary velocity strength) until further downstream. Increased mixing due to the unsteady effects dampens the organized motions of the secondary vortices.

Figure 12 describes the downstream development of the normalized maximum longitudinal velocity at different $R e$. Lower $R e$ are characterized by a more developed profile, since the value of $U_{1}^{*}$ is closer to 2 , which is the value 


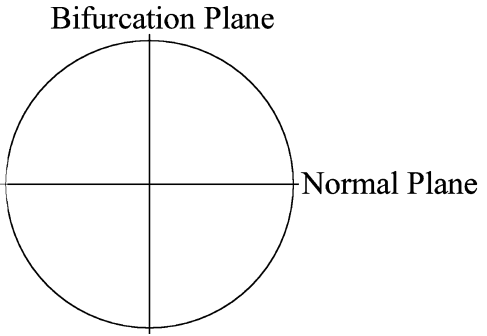

$5 \mathrm{~mm} / \mathrm{s}$

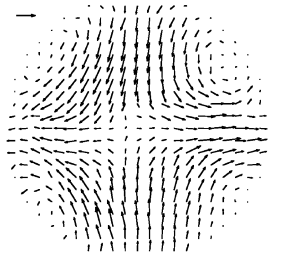

$\mathrm{x} / \mathrm{D}=0$

(a)

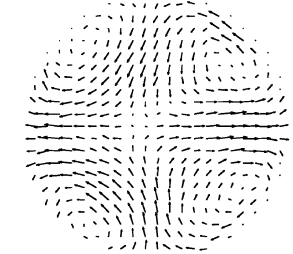

$\mathrm{x} / \mathrm{D}=1$

(b)

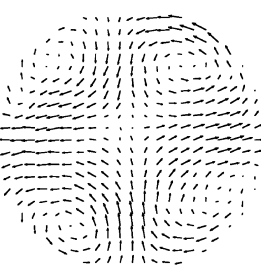

$\mathrm{x} / \mathrm{D}=2$

(c)

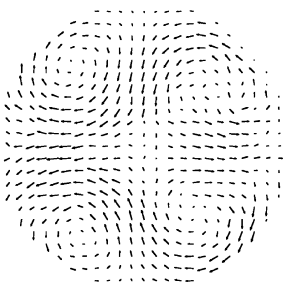

$\mathrm{x} / \mathrm{D}=4$

(e)
Fig. 9. Cross-sectional PIV: $R e=850$

corresponding to fully developed flow in a pipe. The bellshape and double-peak character of the moderate and higher $R e$ is evidenced by a lower value for normalized maximum secondary velocity. All values of $U_{1}^{*}$ are relatively constant for a given $R e$ as the flow progresses downstream, implying a constant mass flux in the longitudinal direction of the bifurcation plane. The mass injected by the secondary currents into the primary direction is equal to the amount of primary mass flux lost to the secondary flow. This argument suggests a constant exchange between the primary and secondary flows.

The evolution of the normalized maximum radial velocity is illustrated in Fig. 13. Radial velocity reaches a maximum at $x / D=0$ and quickly decreases downstream. Past researchers (Zhao and Lieber 1994; Zhang and Kleinstreuer 2002) have shown a similar trend. The radial inrush at the junction can be attributed to the angles at which the daughter branches intercept the parent. At further positions downstream, the radial velocity is minimal for all Re.

\section{4}

\section{Conclusions}

Experiments were performed to quantify in detail the secondary flows during expiration in a symmetric

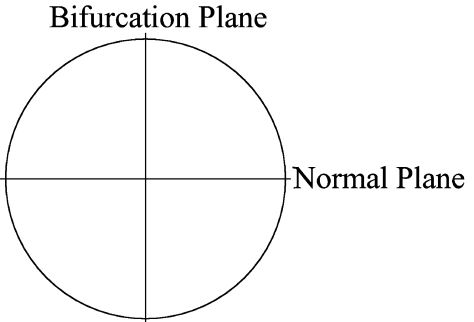

$5 \mathrm{~mm} / \mathrm{s}$

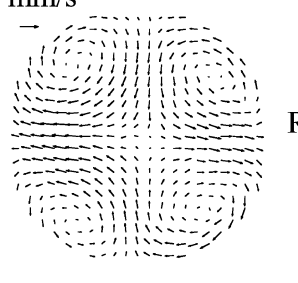

$\mathrm{Re}=680$

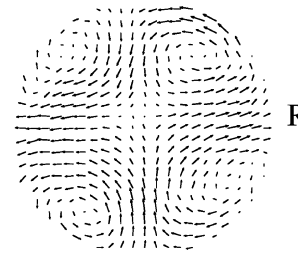

$\mathrm{Re}=850$

(a)

(b)

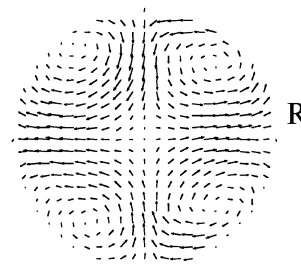

$\mathrm{Re}=1020$

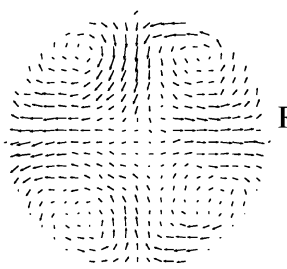

$\mathrm{Re}=1200$

(c)

(d)

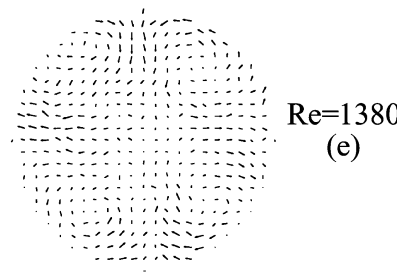

Fig. 10. Cross-sectional PIV: $x / D=2$

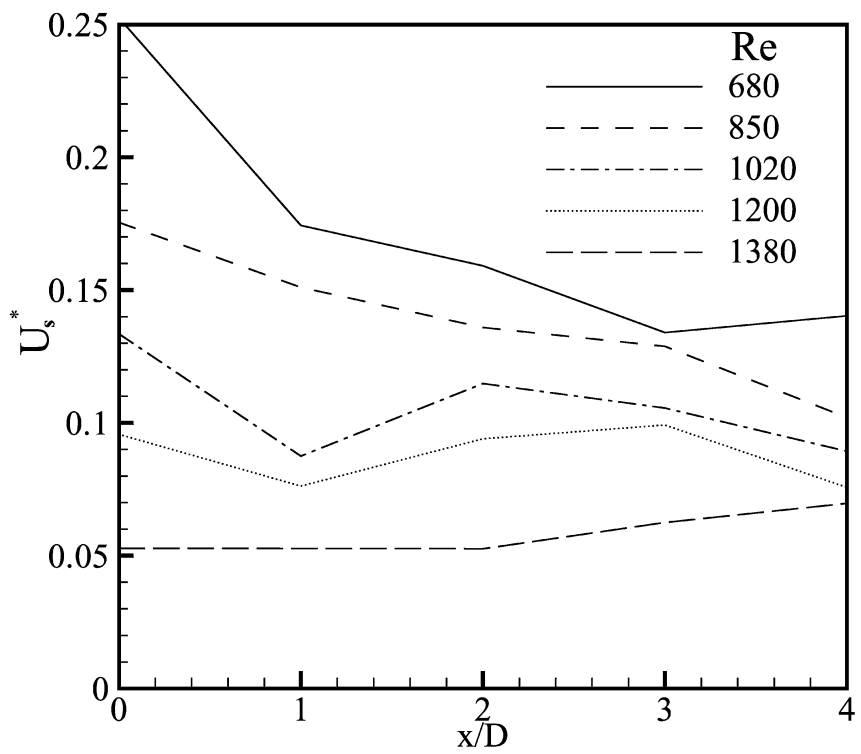

Fig. 11. Downstream development of normalized maximum secondary velocity

bifurcation. Results agree with past studies and also reveal new features. The evolution of dye was documented over a range of injection positions and $R e$. Asymmetries and 


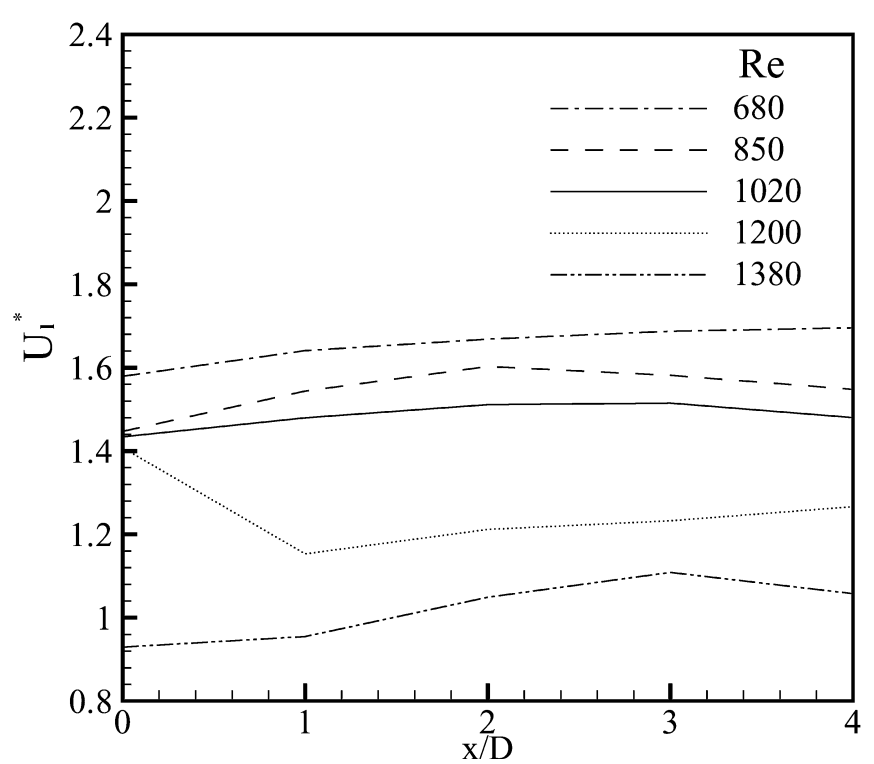

Fig. 12. Downstream development of normalized maximum longitudinal velocity

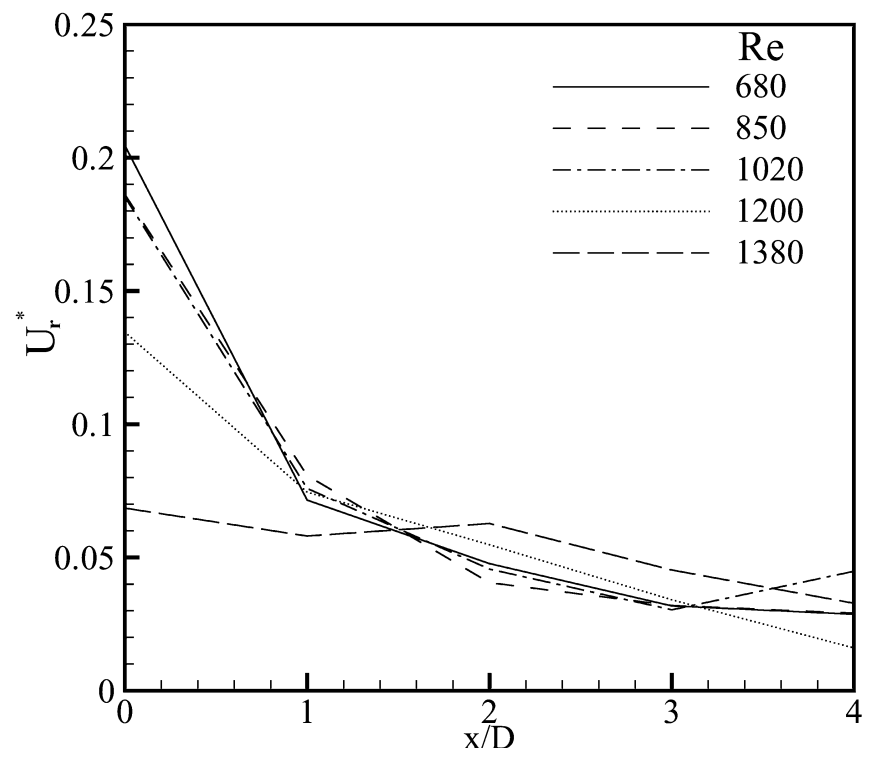

Fig. 13. Downstream development of normalized maximum radial velocity

unsteadiness were discovered, especially at high $R e$. It is especially meaningful to discern that the inside injection was characterized by rapid radial spread while the center injection traversed the bifurcation with little perturbation.
Longitudinal velocity profiles can undergo large differences in the relatively small parameter space of this study. The evolution of the flow from double-peaked, to bell-shaped and finally parabolic as $x / D$ increased or $R e$ decreased was useful. The dependence of the morphology of the bell-shaped profile on secondary velocity strength supports previous studies (Schroter and Sudlow 1969; Zhao and Lieber 1994). Longitudinal PIV also highlights the constant interaction of the primary and secondary flows for the conditions of this study.

Quadruple vortex measurement in cross-sectional PIV experiments is a novel accomplishment. Migration of the vortex cores in the cross-section over a range of $R e$ gives a sense of secondary flow development and clarifies centrifuging regions for particle dispersion. Also, since the maximum secondary velocity was found at the junction, it can be expected that this region will experience the most deposition by inertial impaction.

Most likely due to the model geometry, a hairpin vortex was established which enriched the flow physics. This effect only appeared at higher $R e$, but the interaction with the primary and secondary flow was pronounced.

Unsteadiness due to the hairpin was found in both the LIF and PIV.

\section{References}

Comer CK, Kleinstreuer C, Zhang Z (2001) Flow structures and particle deposition patterns in a double-bifurcation airway models. 1. Air flow fields. J Fluid Mech 435:25-54

Drazin PG, Reid WH (1981) Hydrodynamic stability. Cambridge University Press, New York

Horsfield K, Dart G, Olson DE, Filley GF, Cumming G (1971) Models of the human bronchial tree. J Appl Physiol 31:207-217

Kim CS, Garcia L (1991) Particle deposition in cyclic converging tube flow. Aerosol Sci Technol 14:322-330

Kim CS, Fisher DM, Lutz DJ, Gerrity TR (1994) Particle deposition in bifurcating airway models with varying airway geometry. J Aerosol Sci 25:567-581

Olson DE (1971) Fluid mechanics relevant to respiration: flow within curved or elliptical tubes and bifurcating systems. PhD Thesis, Imperial College, London

Pedley TJ (1977) Pulmonary fluid dynamics. Annu Rev Fluid Mech 9:229-274

Sarangapani R, Wexler AS (1999) Modeling aerosol bolus dispersion in human airways. J Aerosol Sci 30:1345-1362

Schroter RC, Sudlow MF (1969) Flow patterns in models of the human bronchial airways. Respir Physiol 7:341-355

Weibel ER (1963) Morphometry of the lung. Springer, Berlin Heidelberg New York

Zhao Y, Lieber BB (1994) Steady expiratory flow in a model symmetric bifurcation. J Biomech Eng 116:318-323

Zhang Z, Kleinstreuer C (2002) Transient airflow structures and particle transport in a sequentially branching airway model. Phys Fluids 14:862-880

Zhang Z, Kleinstreuer C, Kim CS (2001) Flow structure and particle transport in a triple bifurcation airway model. J Fluids Eng $123: 320-330$ 